\title{
Recognize Symptoms of Disease \& Treatment
}

\author{
Uqbah Iqbal* \\ Department of Physical Disability, Malaysia
}

*Corresponding author: Uqbah Iqbal, Department of Physical Disability, Life Planner, Suite P4, Level 31, AIA Cap Square Tower, Jalan Munshi Abdullah, 50100 Golden Triangle, Kuala Lumpur, Malaysia.

Received Date: November 16, 2018

Published Date: December 05, 2018

\section{Symptoms of Disease \& Treatment}

Written by Prof. Madya Hanafi Ismail and Sadali Othman, this book exposes to readers about the affairs of all kinds of illness, preventative measures and methods of treatment based on current medical disciplines. It is a requirement for everyone to know from the beginning about the causes of the disease, the risk factors, the symptom signs and the treatment and the detection of a disease. It is very important that preventive measures be taken, and actions can be taken immediately. There are four types of illnesses discussed in this book. Lack of blood capability to transport oxygen to tissues in the body can cause anemia. There are several types of anemia, the lack of red blood production due to lack of iron, vitamin B12 and folic acid (folic acid) in the body, excessive cell destruction due to thalassemia disease, G6PD, chemotherapy treatment to prevent cancer, greater cell needs the missing blood cells are not replaced. Symptoms or symptom of anemia are rapidly feeling tired and pale, lethargic and can affect heart function (faster throbbing). Treatment or detection of anemia is a test of hemoglobin and blood (full blood picture) levels.

The arrhythmia occurs when there is an electrical disorder in the heartbeat. These types of disorders are heartbeat too strong, it is actually a powerful heartbeat force (rumble blood) as it receives an external stimulus to pump more energy when someone is scared, too happy or nervous, the body will release the adrenative hormone that stimulates the heartbeat, it is more noticeable to the skinny individual because the heart position is closer to the chest bone, it also happens to individuals suffering from thyroxicosis, lean heart valve and some coronary heart, heart rate is too fast, heart rate 150 minutes without cause is likely to experience heart failure of the heart, it is also known as supraventricular tachycardia stemming from excessive circulation in the heart and those born with this disease, the person will feel sudden palpitations, breathing difficulties, dizziness and body cold sweating, heartbeat is too slow, where the pulse pulses under 60 beats per minute without a reason, the electric stimulus may not be perfectly upright, it often occurs in elderly and coronary heart patients where the wiring circuit is somewhat obsolete, blood will not reaching the brain perfectly where the patient usually gets dizzy or faint and is also known as heart block. Symptoms or symptom is a sudden throbbing chest or sudden throbbing, an attack occurs several minutes, hours or days, the sudden interruption of the tumor, seems to urinate during chest invasion, dizziness, nausea and chest pain during the attack, fatigue after an attack, the heart is stalled, dizziness and fainting occur repeatedly. Treatment or detection of arrhythmia is chest x-ray, ECG and physiological electrophoresis.

Arteriosclerosis occurs when the arteries become narrower due to the accumulation of fat on the wall and the blood transfusion to the other organs of the body will be not smooth. Types of illnesses are arteriosclerosis processes that occur along with the age of humans and secondary types occur as a result of other diseases such as high blood pressure and diabetes. The cause or risk of arteriosclerosis disease is unhealthy eating and less exercise activity. Symptoms or symptom arteriosclerosis is easily tired, and the body is always weak. Prevention of arteriosclerosis is a reduction in fatty foods, avoid poor cholesterol (low density dipoprotein), eat more vegetables and fruits, avoid smoking, do exercise and do not drink liquor. Complications of arteriosclerosis are high blood pressure (hypertension), stroke, heart disease, weak heart, kidney failure, vascular peripheral disease involving foot pain and obscurity and eye damage.

Heart disease occurs when the heart experiences various problems such as disorders, infections, damage and blockages which eventually result in failure to function perfectly. The heart has four valves. Heart valve disease occurs when the valves can become narrow or leaky. The heart valve is a part that is always harmed by thematic fever disease. Congenital heart disease occurs during the birth. The wall of the heart that is not properly closed causes the heart to be hollow. When the heart muscle is damaged, the heart function as a pump will be disrupted. Diseases that often damage the heart muscle are high blood pressure, viral attacks and some of its unknown causes. The heart has natural wiring, throbbing at 
the beat that receives direct stimulation from the nervous system and the hormone in the blood. Abnormalities in the wiring system, rhythmic damage or heart muscles cause the heart to throbbing too fast or slowly. Coronary heart disease occurs when the coronary vessels that carry blood into the heart are smooth and do not have any of these layers becoming thicker and narrow. This narrowing occurs because fat or cholesterol in the blood is attached to the wall of the vessel. It makes it harder for blood to flow into the heart muscles. The implication is that ischemia where the heart is lacking in oxygen and energy to work and causes a person to feel chest pain. If it happens suddenly the blood supply to the heart will be cut off. It is known as heart attack and the patient will die suddenly.

Having a heart disease or risk factor is a smoker aged between 30-40 years, persistent alcohol consumption, high fatty foods or high cholesterol diet and a poorly clean environment. Symptoms or symptom of heart disease are chest pain like something pressing, this pain is then spread to the neck, left hand, pain or pain as if it burns, breathing difficulties and sweating and feeling palpitations. Prevention of heart disease is a healthy and balanced diet, exercise appropriate exercise and remove sweat and get a doctor's examination when you are 40 years old and above. Complications of heart disease is that if heart failure is not detected in the early stages of the patient will find sudden death. In terms of treatment or detection of heart disease, heart disease can be afflicted by all ages, so early treatment is necessary when the patient complains often faint and chest pain.

\section{Acknowledgement}

None.

\section{Conflict of Interest}

No conflicts of interest. 